[Agr. Biol. Chem., Vol. 36, No. 5, p. 875 876, 1972]

\title{
On the Acaciin
}

\author{
By Shintaro KAMIYA, Sachiko ESAKI and Misao NAKA \\ Food Chemistry Laboratory, Shizuoka Women's College, Japan \\ Received October 14, 1971
}

In 1925, Hattori $^{1 /}$ isolated acacetin-7-diglycoside (I), $m p 260^{\circ} \mathrm{C}$, from the leaves of Robinia pseudoacacia L. and proposed to name it acaciin. He considered that the compound I has the structure of acacetin-7-dirhamnoside from some experimental data. Up to the present, several workers have studied on the isolation of flavonoid glycosides in the leaves of the same plant. In 1950, Zemplén and Mester ${ }^{2 !}$ found acacetin-7-rhamnoglucoside, $\mathrm{mp} 263^{\circ} \mathrm{C}$, which was thought to be identical with acaciin. Subsequently, Freudenberg and Hartmann $^{3 /}$ and also Kubota and Hase ${ }^{4}$ isolated acacetin rhamnoxyloglucoside (II), mp $262 \sim 263^{\circ} \mathrm{C}$, as main product and less amount of acacetin rhamnoglucoside, $\mathrm{mp} 262 \sim 263^{\circ} \mathrm{C}$, which they designated as acaciin. Recently Wagner et al. ${ }^{5}$ have decided that acacetin-7rhamnoglucoside is identical with linarin in all respects and further mentioned that since acaciin is thought to be identical with linarin, though no direct comparison with original acaciin was made, the name acaciin should be deleted from the literature hereafter.

On the other hand, the present authors were presented original acaciin (I) isolated by Hattori through the courtesy of Prof. Shimokoriyama and had an opportunity to reinvestigate its properties.

$R f$ value and IR spectrum of the compound I were considerably different from those of

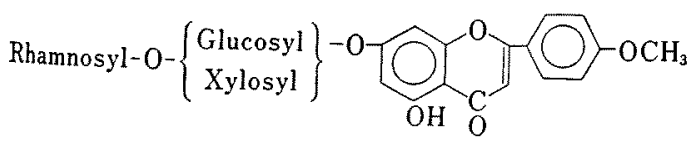

FIG. 1. Chemical Structure of Acaciin. linarin, respectively. On the complete hydrolysis with acid it yielded acacetin, glucose, xylose and rhamnose while on the partial hydrolysis with mold rhamnosidase $e^{6,71}$ it yielded rhamnose and acacetin-7-xyloglucoside. Hence, the original acaciin is thought to have the structure of acacetin-7-rhamnoxyloglucoside as shown in figure, and is identical with the compound II. Accordingly, the name acaciin should be preserved in the literature as before. We could also isolate acaciin as principal product from the leaves of $R$. pseudoacacia L. collected in Shizuoka city.

\section{EXPERIMENTAL}

Measurements of the spectra. IR spectrum was recorded on a JASCO Model IRA-1 spectrophotometer. A Beckman DB recording spectrophotometer was used for UV spectrum.

Paper chromatography. Flavonoids were developed in the following solvent systems (1) butanol: acetic acid: water (4:1:2) and (2) acetic acid: water $(6: 4)$ and detected by spraying diazotized sulfanilic acid followed by sodium carbonate (200) solution. Sugar components were irrigated in the solvent system (1) and detected by spraying aniline-phthalic acid reagent.

Characterization of the original acaciin (I).

$\operatorname{mp} 260^{\circ} \mathrm{C}$.

$\mathrm{UV} \lambda_{\max }^{\mathrm{EtOH}} \mathrm{m} \mu: 265,321 ; \lambda_{\mathrm{max}}^{\mathrm{EtOH}-\mathrm{MeONa}{ }^{81}} \mathrm{~m} \mu: 286,356 ;$

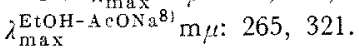

$R f$ value: 0.29 (solvent 1); 0.87 (solvent 2). For reference, $R f$ values of linarin were 0.42 (solvent 1) and 0.70 (solvent 2), respectively.

Anal. Calcd. for $\mathrm{C}_{33} \mathrm{H}_{40} \mathrm{O}_{18} \cdot 2 \mathrm{H}_{2} \mathrm{O}$ (acacetin-7-rhamnoxyloglucoside) $\mathrm{C}, 52.10 ; \mathrm{H}, 5.83 \%$. Found. C, $51.90 ; \mathrm{H}, 6.050^{\circ}$.

Complete hydrolysis of the compound $I$. The com- 
pound I ( $7 \mathrm{mg}$ ) suspended in $2.5 \mathrm{ml}$ of sulfuric acid $(5 \%)$ was refluxed for $30 \mathrm{~min}$ and after cooling the reaction mixture was neutralized with barium carbonate and filtered. The filtrate was evaporated in vacuo to a small quantity and subjected to paper chromatography. As the result, rhamnose, xylose and glucose were detected. Judging from the size and color density of the spots, three sugars seem to be contained in the same ratio.

Enzymatic partial hydrolysis of the compound 1 . The rhamnosidase solution was prepared according to the procedure already reported.6,7) To a solution of the compound I (10 mg) in water $(40 \mathrm{ml}, \mathrm{pH} 6.8)$ was added rhamnosidase solution $(7 \mathrm{ml})$ and the mixture was kept for $2 \mathrm{hr}$ at $40^{\circ} \mathrm{C}$. After the reaction was over, the solution was concentrated in vacuo to a small quantity and then subjected to paper chromatography. As the result, rhamnose and acacetin-7xyloglucoside ( $R f 0.60$ by solvent 1) were detected.

Acknowledgement. We are very grateful to Prof. M. Shimokoriyama of Tokyo University and Prof.
T. Nakabayashi of Shizuoka University for the kind supply of acaciin.

\section{REFERENCES}

1) S. Hattori, Acta Phytochim., 2, 99 (1925).

2) G. Zemplén and L. Mester, Magyar K'miai Folyoirat (Hungary), 56, 2 (1950).

3) K. Freudenberg and L. Hartmann, Ann., 587, 207 (1954).

4) T. Kubota and T. Hase, Nippon Kagaku Zasshi, 87, 1206 (1966).

5) H. Wagner, G. Aurnhammer, L. Hörhammer and L. Farkas, Chem. Ber., 102, 1455 (1969).

6) S. Kamiya, Memoirs of Shizuoka Women's College, 8,9 (1962).

7) S. Kamiya, S. Esaki and M. Hama, Agr. Biol. Chem., 31, 133 (1967).

8) T. J. Mabry, K. R. Markham and M. B. Thomas, "The Systematic Identification of Flavonoids," Springer-Verlag, 1970, p. 41. 